\title{
Can IBEX detect interstellar neutral helium or oxygen from anti-ram directions?
}

\author{
A. Galli and P. Wurz \\ Physics Institute, University of Bern, Bern, 3012, Switzerland \\ J. Park, H. Kucharek, E. Möbius, and N. A. Schwadron \\ University of New Hampshire, Durham, NH 03824, USA \\ J. M. Sokół, M. Bzowski, M. A. Kubiak, and P. Swaczyna \\ Space Research Centre, Polish Academy of Sciences, Warsaw, 00-716, Poland \\ and \\ S. A. Fuselier and D. J. McComas \\ Southwest Research Institute, San Antonio, TX 78228, USA \\ University of Texas, San Antonio, TX 78249, USA
}

Received — 


\begin{abstract}
To better constrain the parameters of the interstellar neutral flow we searched the IBEX-Lo database for helium and oxygen from the interstellar medium in the anti-ram direction in the three years (2009-2011) with the lowest background rates. We found that IBEX-Lo cannot observe interstellar helium from the antiram direction because the helium energy is too low for indirect detection by sputtering off the IBEX-Lo conversion surface. Our results show that this sputtering process has a low energy threshold between 25 and $30 \mathrm{eV}$, whereas the energy of the incident helium is only $10 \mathrm{eV}$ for these observations. Interstellar oxygen, on the other hand, could in principle be detected in the anti-ram hemisphere, but the expected magnitude of the signal is close to the detection limit imposed by counting statistics and by the magnetospheric foreground.
\end{abstract}

Subject headings: ISM: atoms (He and O) ISM: clouds ISM: kinematics and dynamics methods: data analysis solar neighborhood Sun: heliosphere 


\section{Introduction}

The Interstellar Boundary EXplorer (IBEX, McComas et al. 2009) directly measures the inflow of interstellar neutral (ISN) matter into the heliosphere. Inverting the strength and location of the signal from the observed neutral species (mainly helium, hydrogen, and oxygen) allows us to determine the flow velocity, temperature and direction of interstellar matter with respect to the heliosphere (Möbius et al. 2009). So far, only observations from ram-directions when IBEX is moving toward the inflow direction (January through March) showed a clear interstellar signal. From these so-called "spring peak" observations, a narrow tube of coupled ISN flow parameters was derived, with the flow latitude, speed, and temperature depending on the flow longitude (Bzowski et al. 2012; Möbius et al. 2012; McComas et al. 2012) and a relatively large uncertainty along the tube. An observation of the ISN flow peak in October when IBEX is moving away from the interstellar signal would yield a second, almost orthogonal, parameter tube. This would allow to constrain the parameter range significantly. Figure 1 shows a sketch of the two possible flow peaks, the solid trajectory curve represents the detected spring signal, the dashed curve denotes the so far undetected fall signal. Möbius et al. (2012) studied the ISN spring signal of helium, stating that "attempts to obtain a complementary measurement of the flow direction in the fall (...) have not been successful". This work is a more thorough attempt to obtain a fall measurement by combining several years of data and provide an upper limit for the expected signal. The results are important in at least two respects. Detection or upper observational limits of the fall signal of ISN helium and oxygen may allow us to better constrain the ISN flow parameters. Moreover, this study also constrains the instrument sensitivity to energetic neutral atoms (ENAs) and in particular to sputtering effects below $100 \mathrm{eV}$. This information is needed to better compare models to IBEX-Lo measurements

both for heliospheric ENA studies at low energies (Galli et al. 2014; Schwadron et al. 2014) and for ISN studies (Sokót et al. 2015b). Among the other special issue contributions, 
the most relevant for the search of the fall signal are: Sokót et al. (2015a) describing the numerical model whose results are used here, Sokót et al. (2015b) presenting the details of the expected helium fall peak, Park et al. (2015) presenting global maps of heavy elements, and Kucharek et al. (2015) quantifying gravity disturbances on the ISN signals.

In Section 2 we present the data selected for this study and explain our methods to derive maps of the ISN signal. In Section 3 we calculate the expected intensity and location of the ISN fall signal. Section 4 compares the predictions with observations, and Section 5 concludes the paper.

\section{Dataset}

This section deals with the selection and the processing of IBEX-Lo data to create maps of the ISN signal for ram and anti-ram directions. The distinction between ram and anti-ram observations is important for the analysis because IBEX moves with Earth on its orbit at a speed of roughly $30 \mathrm{~km} \mathrm{~s}^{-1}$ relative to the heliosphere. During a ram observation, IBEX is moving toward the observed ISN inflow. Eight months later, IBEX images the other branch of the ISN around the Sun while moving away from the inflow (see Fig. 1). The observed intensity and energy of the interstellar neutrals is notably higher for ram than for anti-ram observation because the velocity of the interstellar flow at $1 \mathrm{AU}$ in the solar reference frame $\left(50 \mathrm{~km} \mathrm{~s}^{-1}\right)$ is not much larger than the relative velocity of IBEX and the Earth around the Sun $\left(30 \mathrm{~km} \mathrm{~s}^{-1}\right)$. The velocity of the ISN atoms with respect to IBEX reaches $50+30 \mathrm{~km} \mathrm{~s}^{-1}$ for ram observations in February and March, whereas it is only $50-30 \mathrm{~km} \mathrm{~s}^{-1}$ for anti-ram observations in October. More generally, the velocity $v_{m}$ in the IBEX reference frame depends on the inertial velocity $v_{i}$ via the proper motion of the spacecraft $u_{s c} \approx 30 \mathrm{~km} \mathrm{~s}^{-1}$ and the latitude or spin angle $\theta_{m}$ from which the signal appears 
to originate in the spacecraft reference frame (McComas et al. 2010):

$$
v_{m}=u_{s c} \sin \left(\theta_{m}+180^{\circ}\right)+\sqrt{v_{i}^{2}+u_{s c}^{2}\left(\sin ^{2}\left(\theta_{m}+180^{\circ}\right)-1\right)} .
$$

Equation 1 yields a maximum of $v_{m}=80 \mathrm{~km} \mathrm{~s}^{-1}$ for the ram observations at $\theta_{m}=270^{\circ}$ and a minimum of $20 \mathrm{~km} \mathrm{~s}^{-1}$ for the anti-ram observations at $\theta_{m}=90^{\circ}$. We call the first signal the spring peak or spring signal, the second signal, which has not been detected so far with IBEX, is called the fall signal.

The IBEX-Lo sensor is an ENA camera, sampling neutral atoms in eight energy bins between 10-2000 eV (Fuselier et al. 2009). For a neutral atom to trigger a signal in IBEX-Lo, the particle must either be negatively charged when it is reflected off the conversion surface, or it must sputter a negative ion from the conversion surface (Wurz et al. 2008; Rodriguez et al. 2015). Whereas oxygen atoms have a high probability of forming negative ions, neutral helium atoms can be detected in IBEX-Lo only via surface sputtering of hydrogen ions. After conversion, the negative ions are pre-accelerated into a scanning electrostatic analyzer that defines the different energy bins. Table 1 lists the center energies and lower and upper half widths at half maximum for all IBEX-Lo energy bins. The three lowest energy bins (bin 0.5, 1, and 2) are most interesting for this work because they cover the velocities associated with ISN helium and oxygen from the anti-ram direction. After the electrostatic analyzer, the ions are post-accelerated to a final energy of $16 \mathrm{kV}(7 \mathrm{kV}$ after summer 2012) and analyzed in an attached time-of-flight mass spectrometer with two carbon-foils between the electrostatic analyzer and the micro-channel plate. This setup yields for each particle the three times-of-flight TOF0, TOF1, and TOF2. They denote, respectively, the time-of-flight of the ion between the first carbon foil and the micro-channel plate, between the second carbon foil and the micro-channel plate, and between the first and the second carbon foil. For this study, we only considered triple coincidence hydrogen and oxygen histograms, i.e., histograms from events with three valid times for TOF0, 
TOF1, and TOF2, whereby TOF0 $\approx$ TOF1 + TOF2. This is the data subset with the lowest noise contribution because it excludes most random single coincidences due to UV light or other sources (Fuselier et al. 2009). The measured time-of-flight is also used to classify the particle as a hydrogen or an oxygen atom. We refer the reader to Rodriguez et al. (2015) in this issue for more details on the usage of time-of-flight information and sputtering processes from noble gas atoms in IBEX-Lo.

During nominal IBEX-Lo operations, the electrostatic analyzer cycles through each of the energy bins from 1 to 8 (see Table 1) at 60 different $6^{\circ}$ spin angles. This covers one swath of $6.5^{\circ}$ width across both hemispheres of the sky (Fuselier et al. 2009). After an accumulation time of roughly 920 seconds, the data block is written to memory and the next data block is started. This provides an accumulation time per data block of only $920 /(8 \times 60)=1.9$ seconds per energy and per spin angle bin of $6^{\circ}$. During orbits $95-101$ (fall 2010) and 143-144 (fall 2011) IBEX-Lo was operated in special ISN fall settings. In 2010, the electrostatic analyzer settings were modified to sample neutrals with a center energy of $10 \mathrm{eV}$ instead of the nominal $15 \mathrm{eV}$. During these orbits no data at higher energy were sampled, the accumulation time per data block at this energy was therefore eight times longer. In fall 2011 the sensor was operated in a similar mode where energy bin 2 was sampled eight times instead of stepping through the eight different energy bins to concentrate on oxygen from anti-ram directions.

We restricted the data set for this study to the first three fall seasons from October to December in 2009, 2010, and 2011: orbits 49-58 (fall 2009), orbits 95-106 (fall 2010), and orbits 143a-150a (fall 2011). In earlier orbits, IBEX remained completely inside the bow shock of Earth's magnetosphere, which creates an intense signal at low energies (see Section 4). IBEX observations thus can be used to study Earth's magnetosphere (McComas et al. 2011; Petrinec et al. 2011), but for studies on the ISN inflow the magnetosphere presents a 
source of contamination that must be minimized. Later fall seasons need not be considered: the instrument sensitivity was lower because the post-acceleration of IBEX-Lo had to be changed in July 2012 and the background caused by the terrestrial magnetosphere increased in later years as the solar cycle neared its maximum. This is demonstrated in Fig. 2 by the time-series of uncorrelated TOF2 count rates, which are a proxy for the magnetospheric background.

To assimilate the IBEX-Lo data into maps, we filtered the data of hydrogen and oxygen counts in a similar way as for heliospheric ENA maps (Schwadron et al. 2014; Galli et al. 2014). We combined the list of good observation times for heliospheric signals with the requirement that hydrogen ENA counts in energy bins 7 and 8 must not exceed 3 counts per sky hemisphere and data block (Fuselier et al. 2012). Compared to Galli et al. (2014), the only difference in data selection was an additional quality criterion to reduce magnetospheric contamination. This is much more important than for studies of the spring ISN signal because the fall signal of helium is expected to be at least two orders of magnitude less intense (Sokót et al. 2015b). The additional criterion was obtained by observing the average rate of TOF2 counts during quiet time periods in all angular bins outside the ISN inflow. The average rates of TOF2 counts in energy bins 4 and 8 from the quiet time period were multiplied by 1.6 and set as thresholds. Any data block with a TOF2 rate exceeding the threshold in energy bin 4 or 8 was then excluded from analysis. Both hydrogen and oxygen data were culled with this method. A review of oxygen maps and the method how to create them is given by Park et al. (2015). For the special mode data in fall 2010 and 2011, the filter methods based on hydrogen ENA counts and TOF2 counts could not be applied because there were no simultaneous IBEX-Lo data at higher energies. Since statistics were 8 times better for these special mode data, we used the time-series of count rates of the only available energy to exclude data blocks with anomalously high count rates. 
We defined the threshold as

$$
c_{\text {limit }}=\lceil\lambda+3 \sqrt{\lambda}\rceil,
$$

assuming that the count rates were Poisson distributed with $\lambda$ denoting the mean and variance estimated from $N$ data blocks. We verified that this culling criterion leads to similar count rates for similar observations performed in special mode and nominal mode. In fall 2011, IBEX-Lo measured only energy bin 2 during orbits 143 and 144 with an eight times higher duty cycle. In orbits 145 and later, nominal operations were resumed. The average count rates over the anti-ram hemisphere in energy bin 2 calculated to $0.011 \pm 0.005$ cnts $\mathrm{s}^{-1}$ both for the nominal mode and for the special mode culled according to Equation 2.

Unlike the heliospheric ENA maps presented in Galli et al. (2014), the maps in this paper show the measured signal with as few corrections as possible. We did not attempt to correct the measurements for survival probability or to transform them into inertial frame. These effects were included in the numerical forward model whose results can then be directly compared to the observed maps. The strength of any observed signal will be indicated in raw count rates $\left(\mathrm{cnts} \mathrm{s}^{-1}\right)$. They represent the original number of counts per orbit and spin bin, corrected only for accumulation time. Other effects such as throughput effects, sputtering, or energy-dependent conversion efficiencies were disregarded. The last assumption is not entirely true, since the conversion efficiency of hydrogen is three times lower in energy bin 1 than in energy bins 3, 4, and 5. For oxygen atoms, conversion efficiencies in the lowest energy bin are two times lower than in energy bins 5 and 6 . This means that the expected signal from anti-ram directions in the lowest energy bin will be two (for ISN oxygen) or three times (for ISN helium) weaker than in the model (Sokót et al. 2015b). This may introduce a bias when we want to compare the relative magnitude between different energies, but it is irrelevant for discussing map features because the correction factors are constant with time and viewing direction from 2009 to 2011. The count rates were mapped in a rectangular grid, where the $x$-axis is given by the orbit 
number and the $y$-axis is given by the spin angle of the spacecraft. The 8 th orbit of each fall season map is positioned at $\lambda_{e c l}=295^{\circ} \pm 3^{\circ}$. The 30 spin angle sectors between $0^{\circ}$ and $180^{\circ}$ cover anti-ram observations while the hemisphere between $180^{\circ}$ and $360^{\circ}$ covers ram observations.

Galli et al. (2014) quantified IBEX-Lo's ubiquitous background in hydrogen ENA measurements, which depends only on energy bin. The current study has allowed us to re-examine these values and their uncertainties. Table 2 contains the updated estimates, which will also be used in the results section to interpret the observations. The background estimates changed relative to the previous values (Galli et al. 2014) by less than 10\%, but the variability of the background in the two lowest energy bins turned out to be larger (see error bars in Table 2). The ubiquitous background in oxygen maps is ten times lower than the hydrogen background in each of the energy bins 1 to 5 (Park et al. 2015).

\section{Model predictions}

In this section we summarize the expectations regarding the signal of ISN helium and oxygen from the anti-ram hemisphere. We will use simulation results of ISN helium modeled by Sokót et al. (2015b) to estimate by which factor the maximum fall peak will be weaker than the observed spring peaks. An important free parameter in that model is the

energy below which neutral helium can no longer be detected with IBEX because of too low sputtering efficiencies. We therefore first summarize our knowledge of sputtering processes relevant for IBEX measurements. 


\subsection{Energy threshold of sputtering by neutral helium and oxygen}

The two main species constituting the ISN signal near Earth are neutral helium and oxygen. Neutral hydrogen, the dominant component in the ISN outside the heliosphere, can be observed with IBEX-Lo (Rodriguez et al. 2013; Saul et al. 2013; Schwadron et al. 2013) but is mostly depleted at 1 AU due to re-ionization and radiation pressure (Kubiak et al. 2013). IBEX-Lo can detect and identify neutral hydrogen and oxygen atoms based on the time-of-flight signature of an event. Whereas ISN oxygen can be directly measured, ISN helium can only be measured indirectly via sputtering (see Section 2). If the impacting atom is energetic enough, impurities on the conversion surface, water in particular, can be sputtered from the surface and produce a secondary ion signal. The most frequent sputtering reaction is the $\mathrm{He} \rightarrow \mathrm{H}$ reaction. It makes the ISN helium spring peak visible in IBEX-Lo as an apparent hydrogen signal in the four lower energy bins. The measured count rates of this signal from 2010 February are listed in Table 3. At the energies considered here (10-100 eV), only direct knock-off sputtering (sometimes also called recoil sputtering or elastic collision sputtering) needs to be considered, as contributions from reflected ions and cascade sputtering become relevant only at higher energies (Taglauer 1990).

We ran SRIM (The Stopping and Range of Ions in Matter (Ziegler et al. 1985)) simulations for a $10 \AA$ thick layer of water molecules (with a surface binding energy of $2 \mathrm{eV}$ ) on a carbon surface being sputtered by $134 \mathrm{eV}$ helium atoms and $534 \mathrm{eV}$ oxygen atoms to simulate the ISN signal observed from ram directions $\left(v=80 \mathrm{~km} \mathrm{~s}^{-1}\right)$. We then repeated these simulations for lower energies with 10-200 eV helium and oxygen atoms to cover relative velocities representative for anti-ram observations. The results are given in sputter yield, i.e., the ratio of sputtered versus impacting atoms. Figure 3 shows the simulated curves of the four sputter yields for $\mathrm{He} \rightarrow \mathrm{H}$ (black "x"), He $\rightarrow$ O (black circle), $\mathrm{O} \rightarrow \mathrm{O}$ (red circles), and for $\mathrm{O} \rightarrow \mathrm{H}$ (red " $\mathrm{x}$ "). The dashed lines indicate the relative 
velocity of ISN helium (black) and oxygen (red) for ram observations in the spring season, the corresponding dash-dotted lines show the velocities of the ISN species for anti-ram observations in the spacecraft reference frame. These simulations imply that helium atoms below $17 \mathrm{eV}$ are invisible to IBEX-Lo because they are not energetic enough to sputter hydrogen or oxygen atoms. For sputtering oxygen atoms, the corresponding energy would be $25 \mathrm{eV}$. This threshold is irrelevant for IBEX-Lo observations because oxygen atoms can be observed directly, and there is no energy threshold for surface ionization (Wurz et al. 2006). More important for expected ISN oxygen observations (see Section 3.3) is the fact that the $\mathrm{O} \rightarrow \mathrm{O}$ sputter yield for ram observations exceeds 1.0 , whereas it is on the order of 0.01 for anti-ram observations.

The actual energy below which neutral helium cannot be detected with IBEX-Lo may be higher than the sputter threshold. It can be estimated as the energy at which so few hydrogen ions are sputtered that the expected signal drops below the detection limit of 1 count per orbit, corresponding to $C_{0}=0.0015 \mathrm{cnts} \mathrm{s}^{-1}$ :

$$
Y\left(E_{f}\right) / Y\left(E_{s}\right) f_{C G} C_{s}<C_{0}
$$

Here, $C_{s}$ denotes the strength of the ISN signal in spring $\left(23 \mathrm{cnts} \mathrm{s}^{-1}\right), f_{C G}=0.02$ is the relative reduction of the signal from ram to anti-ram viewing geometry (Sokót et al. 2015b), and $Y$ is the energy-dependent sputter yield. Equation 3 yields a minimum $Y\left(E_{f}\right)>0.002$, which according to Fig. 3 corresponds to $17 \mathrm{eV}$. If we replace $C_{0}$ by the typical background level due to magnetospheric background and other noise sources $\left(0.01 \mathrm{cnts} \mathrm{s}^{-1}\right), Y\left(E_{f}\right)$ in Equation 3 increases to 0.011 and the energy threshold shifts to $22 \mathrm{eV}$. We therefore do not expect to observe ISN helium from the anti-ram direction near the ecliptic plane. The speed relative to IBEX $\left(20 \mathrm{~km} \mathrm{~s}^{-1}\right)$ is slower than the $32 \mathrm{~km} \mathrm{~s}^{-1}$ implied by the threshold of $22 \mathrm{eV}$.

The SRIM simulations in this section served to give us an estimate but the relevant 
sputter threshold for observations in space can only be derived from the IBEX observations themselves (see results in Section 4). During calibration of the sensor in the MEFISTO facility of the University of Bern (Fuselier et al. 2009) we did not encounter any threshold for helium sputtering hydrogen between 10 and $100 \mathrm{eV}$. The layer on the conversion surface of IBEX in space is an agglomerate of contaminants (water being the most likely constituent) that cannot be directly reproduced in laboratory. In the laboratory, outgassing of the instrument continued for typically one week, wheres in space it continues for years. Moreover, the total gas pressure at the conversion surface and thus the replenishment rate of the contamination layer was higher by 1 to 2 decades compared to measurements in space.

\subsection{The expected fall signal of ISN helium}

We relied on the analytic Warsaw Test Particle Model (Sokót et al. 2015a) to predict the fall signal of ISN helium. The results of this model and their implications for ISN helium observations are discussed in detail in this issue by Sokót et al. (2015b). The modeled signal of ISN helium is the sum of the primary population (inflow parameters according to Bzowski et al. 2012) and the Warm Breeze (Kubiak et al. 2014), which dominates the ISN observations in November and December at ecliptic longitudes $120^{\circ}-170^{\circ}$. The model code

integrates the trajectory of neutral gas in the inertial frame of the moving spacecraft, taking into account the actual spacecraft velocity relative to the Earth and the Earth velocity relative to the Sun. Changes in the ionization rates inside the heliosphere are taken into account, whereas gravitational effects of the Earth and other planets are neglected. We discuss in this section the results for two cases: an ideal case with energy threshold zero and the realistic case for IBEX measurements where helium atoms below $19 \mathrm{eV}$ become invisible due to the sputtering threshold (see previous Section 3.1). 
Figure 4 shows maps of the predicted ISN helium signal in count rates in the spacecraft reference frame for ram and anti-ram directions. The helium signal was modeled as a combination of the ISN primary population and of the Warm Breeze (adapted from Fig. 7 in Sokót et al. (2015b)). The maps cover one year from 2009 September (orbit 43) until 2010 September (orbit 91). The main signal around orbit 65 is the spring peak from ram-direction, the less intense spot around orbit 50 is the fall peak from anti-ram direction. The maximum of the fall peak is shifted $30^{\circ}$ to the North because of the flux transformation from the solar to the spacecraft reference frame. The calculations yielded intensities, which were integrated from 0 to maximum energy of the ISN signal and averaged over the collimator field-of-view. For this study, we transformed the simulation output into expected count rates by scaling the maximum pixel of the spring peak to the $23 \mathrm{cnts} \mathrm{s}^{-1}$ observed in orbit 64 in 2010 February (see Table 3). The same logarithmic color scale will be used throughout the paper for all predicted and observed maps unless otherwise stated. This logarithmic scale has steps at 0, 0.0002, 0.0015, 0.003, 0.01, 0.03, 0.1, 0.3, and $1.0 \mathrm{cnts} \mathrm{s}^{-1}$. The steps of the color scale were chosen to reflect critical limits for IBEX-Lo observations. First, signals weaker than $0.0002 \mathrm{cnts} \mathrm{s}^{-1}$ cannot be observed, as this rate corresponds to one single count per entire orbit in special fall mode. Second, the integration time per energy bin and pixel in nominal mode is eight times shorter, which leads to a statistical detection limit of $0.0015 \mathrm{cnts} \mathrm{s}^{-1}$. Finally, real observations are not only limited by statistics but also by background sources. The average background in the two lowest energy bins is roughly $0.01 \mathrm{cnts} \mathrm{s}^{-1}$ (see Table 2 ).

The upper panel in Fig. 4 represents the ideal case where no energy threshold for sputtering exists, the lower panel shows a more realistic case where helium atoms below $19 \mathrm{eV}$ (based on the sputtering simulations in Section 3.1) do not sputter hydrogen atoms and thus do not produce a signal in IBEX-Lo. The ratio of maximum fall to spring signal in the upper panel of Fig. 4 is 0.02 , which implies a maximum fall signal of a few 0.1 
cnts $\mathrm{s}^{-1}$ in the absence of an energy threshold for sputtering (Sokót et al. 2015b). The fall peak would appear in orbit 50 in 2009 October in the anti-ram hemisphere at $\lambda_{e c l}=295^{\circ}$ $\beta_{\text {ecl }}=+30^{\circ}$. If, on the other hand, the $19 \mathrm{eV}$ threshold for helium sputtering applies, the fall peak vanishes almost completely from the anti-ram hemisphere, with only two spots remaining close to the poles (spin angle $180^{\circ}$ is situated at the ecliptic North pole). Earth's gravity would shift the expected maximum of the fall signal to an earlier time. The effect amounts to roughly $4^{\circ}$ in longitude for any ISN species (Kucharek et al. 2015). This implies the maximum probably would occur in orbit 49 rather than orbit 50. This effect was not included in the present simulations since it does not affect our conclusions regarding the ISN helium and oxygen in fall.

For any observation, the energy range of IBEX-Lo must be considered because a potential signal in the ecliptic plane would have only $8.4 \mathrm{eV}$ from the anti-ram direction (neutral helium with a relative speed of $20 \mathrm{~km} \mathrm{~s}^{-1}$ ). We therefore added lines to the maps in Fig. 4 to indicate where we would expect the sputtering signals to vanish depending on energy bin. To calculate these latitudes we relied on the energy distribution of the sputtered hydrogen observed in spring: the hydrogen signal in Table 3 appears at equal strength in all energies up to a cut-off at half the energy of the parent helium atoms $(134 \mathrm{eV})$. Above energy bin $3(39-77 \mathrm{eV})$, the sputtering signal decreases by an order of magnitude. We therefore assumed that the sputtering signal also vanishes in any of the three lowest energy bins (see Table 1) wherever the energy of the parent helium atoms drops below twice the lower cut-off of the given energy bin. The apparent helium energy is related to the latitude or spin angle of observations via Equation 1. We found that the signal close to the ecliptic plane is in any case invisible because the apparent helium energies fall below the range of the lowest energy bin. The fall signal appears in the most optimistic case only above $55^{\circ}$ from the ecliptic plane. This is the dash-dotted line in Fig. 4, corresponding to helium atoms faster than $14 \mathrm{eV}$. Signals from these latitudes should be detected only in the special 
fall mode (see Section 2). The dashed lines at a $75^{\circ}$-offset denote the latitude at which the signal should also appear in nominal energy bin 1, i.e., $E_{\mathrm{He}} \geq 22 \mathrm{eV}$. The two solid lines in the ram hemisphere indicate where the sputtering signal also appears in energy

bin $2\left(E_{\mathrm{He}} \geq 40 \mathrm{eV}\right)$. This means that the hydrogen signal produced by ISN helium from anti-ram directions should only be observed in the two lowest energy bins. Moreover, we expect the signature of the Warm Breeze in energy bin 2 only to extend to spin angles $188^{\circ}$ and $352^{\circ}$. These predictions are tested in Section 4 .

\subsection{The expected fall signal of ISN oxygen}

For ISN oxygen, we assumed that oxygen atoms in the heliosphere follow the same trajectories as neutral helium. This can be verified for the spring peak, where IBEX-Lo observes the maximum of $\mathrm{O}, \mathrm{Ne}$, and He at a similar position (Möbius et al. 2009; Rodriguez et al. 2015). To estimate the expected ISN oxygen signal, we took the simulated maps of ISN helium (Sokót et al. 2015b) and scaled the count rates to the peak strength of ISN oxygen observed in 2010 February (see Table 3). This re-scaling corrects for the higher ionization loss rate of oxygen inside the heliosphere compared to helium. The maximum of 0.08 counts $\mathrm{s}^{-1}$ was observed in energy bin 6 whose center energy lies closest to the relative speed of ISN oxygen from ram direction. The signal of sputtered oxygen from helium in the four lower energy bins is irrelevant for anti-ram directions and was therefore excluded from the map. The result is shown in Fig. 5. Apart from the absolute count rates, the shape is identical to the upper panel of Fig. 4 for ISN helium. No low energy threshold needs to be considered for oxygen atoms because no sputtering process is required to detect neutral oxygen with IBEX-Lo. Even oxygen atoms in the ecliptic plane (38 eV energy) should be detectable in energy bin 2 (Wurz et al. 2006). To estimate the magnitude of the signal, we must however consider the energy-dependent contributions of direct oxygen detection and 
oxygen sputtering to the total signal. The spring signal in ram directions was generated by $500 \mathrm{eV}$ oxygen atoms. At these energies, sputtering contributes as many counts to the total signal as direct detection of oxygen atoms (sputter yield of $\mathrm{O} \rightarrow \mathrm{O} \approx 1.0$ in Fig. 3). In the anti-ram hemisphere, the energies of oxygen atoms relative to IBEX are below 200 $\mathrm{eV}$, and the sputter contribution is negligible. We therefore reduced all count rates in the anti-ram hemisphere in Fig. 5 by a factor of 2. The attenuation factor of the fall to spring magnitude thus is 0.01 for oxygen ISN instead of 0.02 (Sokót et al. 2015b), and the oxygen fall signal in energy bins 1 and 2 is expected to reach only 0.0008 counts $\mathrm{s}^{-1}$. The difficulty of finding the fall peak of oxygen in the observations is solely due to these low count rates. The fall signal of ISN oxygen is expected to appear only in the range 0.0002 to 0.0015 cnts $\mathrm{s}^{-1}$ (purple pixels in Fig. 5), which can be resolved only with the special fall mode.

\section{Results}

We concentrate on the two lowest nominal energy bins centered around $15 \mathrm{eV}$ and 29 $\mathrm{eV}$ and the special fall ISN mode centered at $10 \mathrm{eV}$. These are the only energies where we expect signals from ISN helium or oxygen in the anti-ram hemisphere. For comparing the observations with predictions, we assume that the ISN signal does not notably vary from 2009 to 2011. This is reasonable, considering the evolution of ionization rates and survival probabilities given by Bzowski et al. (2013). The simulations by Sokót et al. (2015b) were done for the season $2009 / 2010$.

\subsection{No signature of ISN helium in the anti-ram direction}

We first show in Fig. 6 the hydrogen count rates observed in energy bin 2 for the entire half year from 2009 October to 2010 June (orbits 49-80), including the ram hemisphere 
with the easily visible spring ISN signal. We chose this year for the overview plot because the two later years with fall seasons 2010 and 2011 were not completely covered with identical measurement modes at several energies. The upper panel of Fig. 6 presents the results in the IBEX coordinate system (orbit number versus spin angle), the lower panel presents them in the ecliptic coordinate system, for which we assumed a $6.5^{\circ} \times 6.5^{\circ}$ full-width-half-maximum field-of-view per pixel (Fuselier et al. 2009). Measurements in orbit 49 and earlier are dominated by magnetospheric background. We therefore did not extend the plot farther to the left. The imprint of the magnetosphere in orbit 49 is stronger in the ram hemisphere where the IBEX field-of-view is directed toward the magnetosphere. The Warm Breeze in Fig. 6 is spread out over most of the ram hemisphere, but it vanishes at Northern and Southern latitudes $\left(\lambda_{e c l}= \pm 82^{\circ}\right)$ as predicted in the previous Section 3 for energy bin 2. The reason is that the hydrogen atoms sputtered by helium coming from the Warm Breeze at polar regions have an energy below the lower energy limit of $20 \mathrm{eV}$ of energy bin 2. The anti-ram hemisphere (spin angles $0^{\circ}$ to $180^{\circ}$ in the upper panel) is void of any structure resembling a fall peak signal. Bright stripes in single orbits (orbits 49 and 63) reveal magnetospheric contamination that could not be excluded from the data. The main difference between Fig. 6 and the simulations in Fig. 4 is the ubiquitous background of roughly $0.01 \mathrm{cnts} \mathrm{s}^{-1}$ (not included in the simulations), corresponding to pale blue in our color scale.

Figure 7 shows the complete map of the hydrogen signal in energy bin 1 in the identical format as the upper panel of Fig. 6. The spin angles $6^{\circ}-24^{\circ}$ were omitted because of a constant instrument background in the lowest energy bin at these angles. At this energy (11-21 eV) a major discrepancy with the predictions shows up. From orbit 50 to 55 the signature of the ISN flow should extend from the ram hemisphere down to the dashed line (copied from the map of predicted count rates in Fig. 4) in the anti-ram hemisphere. However, the halo of the Warm Breeze extends only to spin angles $186^{\circ}$ and $354^{\circ}\left( \pm 84^{\circ}\right.$ 
off ecliptic in the ram hemisphere), where it abruptly disappears. The reason is the low energy threshold of sputtering. This was predicted by Kubiak et al. (2014); now we can demonstrate it.

Figure 8 groups all three years of fall data on one page. In contrast to Figs. 6 and 7, only the orbits from October until December are shown, i.e., for the time when we expect the fall peak. Orbits 49-58 correspond to 2009 October 13 until December 24, orbits 97-106 correspond to 2010 October 13 until December 24, and orbits 143-150 correspond to 2011 October 18 until December 24. The left fringe of the plots becomes increasingly bright because IBEX spends longer time inside the bow shock of Earth's magnetosphere. Orbits before October 13 (orbits 49, 97, and 143) were omitted. Because of the special fall modes in fall 2010 (orbits 95-101) and in fall 2011 (orbits 143-144), for 2010 only the map with the lowest energy bin can be completed, for 2011 this applies for energy bin 2. The top row of Fig. 8 shows the results for fall 2009 (this is a close-up of the left part in the previous Figs. 6 and 7), bottom left shows the result for fall 2010, the bottom right shows the results for fall 2011. The red lines were copied from Fig. 4, showing the latitude where the ISN signal is expected to disappear.

From our most sensitive observations in fall 2009 and 2010 we compiled latitudinal profiles of the observed fall signal of sputtered helium and compared them to model predictions with different energy cut-offs. The result is shown in Fig. 9 as count rate profiles versus spin angles from $0^{\circ}$ to $360^{\circ}$, the higher spin angles covering the ram observations. The black " $\mathrm{x}$ " symbols in the top panel represent the average during the special mode observations in fall 2010 in orbits 99-101, measurements obtained in fall 2009 are added as red circles (energy bin 1) and as blue squares (energy bin 2). As mentioned previously, the line profile of energy bin 2 vanishes exactly at those spin angles (blue vertical lines) expected from the finite energy range $(20$ to $41 \mathrm{eV})$ of the instrument. At energy bin 1 , 
the discrepancy between expected cut-off (red vertical lines) and observations becomes apparent. The cut-off differs only by one spin sector from the one found for the special fall mode. The bottom panel of Fig. 9 shows model simulations with three different thresholds for sputtering at 0 (orange dashed line), 19 (green solid line) and $38 \mathrm{eV}$ (purple dashed line).

Fig. 9 demonstrates that the energy threshold of sputtering must be $25-30 \mathrm{eV}$. Inside the anti-ram hemisphere there is no data point that lies more than $2 \sigma$ above the background level (dotted line). The ISN helium signal appears only at the North pole (spin angle = $180^{\circ}$ ) and then rapidly increases toward ram directions. This is two spin sectors later than expected if the sputtering cut-off were $19 \mathrm{eV}$. It happens three spin sectors before the model with an energy cutoff at $38 \mathrm{eV}$. This confirms the sputtering simulations in Section 3.1 that predicted a lower energy threshold around $20 \mathrm{eV}$ for helium atoms sputtering hydrogen. As a consequence, IBEX did not and cannot detect ISN helium atoms in the fall season from anti-ram directions.

The top panel of Fig. 9 also demonstrates that the place of the observed maximum of the ISN helium signal in the ram hemisphere depends on energy. For energy bin 2, the measured profile agrees with model predictions (purple curve) for all spin sectors inside the range defined by the instrument energy response. At lower energies the apparent maximum shifts toward directions where helium atoms of a lower energy originate from. The model predictions with the empirically found sputtering threshold will have to be multiplied by an energy-dependent instrument response function to allow for a more quantitative analysis of the Warm Breeze during the fall season (Sokót et al. 2015b).

The error bars of the data points in Fig. 9 were derived as follows: if the number of counts $N$ included in the average exceeded 20, the error bar of a data point is given by its standard deviation. For very low signals in the anti-ram hemisphere the results 
are limited by statistics and the error bar is given by $\sqrt{N}$ divided by the accumulation time. The absolute calibration error per energy bin of 30\% (Galli et al. 2014) was not considered as it would affect only the comparison of different energy bins. In Fig. 9, the region inside the red dashed lines $\left( \pm 55^{\circ}\right.$ off the ecliptic plane in anti-ram hemisphere) is void of any ISN signal because any sputtered hydrogen would have insufficient energy to be detected in IBEX-Lo. We therefore used this region to establish the background level to be $0.0062 \pm 0.0005 \mathrm{cnts} \mathrm{s}^{-1}$. This is about two third of the ubiquitous background found in the two lowest nominal energy modes. The uncertainty of the background is too small to affect the determination of the energy cut-off. For the two lowest nominal energy bins, a more thorough analysis covering four years of ram and anti-ram observations at $310^{\circ}$ to $360^{\circ}$ ecliptic longitude (i.e., orbits 53 to 58, 101 to 106 etc.) was performed. On average, a background of $0.009 \mathrm{cnts} \mathrm{s}^{-1}$ for the two lowest nominal energy bins was found (see Table 2). These values are representative if we average over four years and several latitudes. Most pixels in fall 2009 at energy bin 1 and 2 had a background level of only 0.005 to 0.007

$\operatorname{cnts~s}^{-1}$. This indicates that the ubiquitous background is the sum of various local sources (largely the magnetosphere), which may be weaker for single orbits, but cannot be removed on a general basis.

\subsection{Results for ISN oxygen - upper limit of the fall signal}

Figure 10 shows the observed oxygen count rates in the fall seasons of 2009 (top panels), 2010 (bottom left) and 2011 (bottom right) in the identical format as Fig. 8 did for hydrogen count rates. The bright halo in ram pixels (spin angle $180^{\circ}-360^{\circ}$ ) is produced by ISN helium that sputters oxygen atoms. At these low energies, this signal dominates over the signal from real ISN oxygen, which is on the order of $0.001 \mathrm{~s}^{-1}$ from October to December (see map of expected oxygen in Fig. 5). This forces us to restrict a direct 
comparison between observed and predicted oxygen maps to those latitudes in the anti-ram hemisphere where we are sure to not see any sputter signal from ISN helium. According to Fig. 8, this leaves all pixels within the dashed line, i.e., spin angles $15^{\circ}$ to $165^{\circ}$. The most obvious difference from the hydrogen count rates discussed in the previous section is the ten-fold decrease in count rate. This reduction applies both to the halo of sputtered oxygen from ISN helium in ram directions and to the background (plus potential fall signal) in anti-ram directions. The average background of oxygen maps was estimated from the average over orbits 53-58 in the anti-ram hemisphere where we neither expect nor see any helium or hydrogen signal (refer to upper panel of Fig. 8). The background calculated was $0.0009 \pm 0.0001 \mathrm{cnts} \mathrm{s}^{-1}$ in energy bin 1 and to $0.0008 \pm 0.0001 \mathrm{cnts} \mathrm{s}^{-1}$ in energy bin 2. The uncertainty was derived from Poisson statistics. During the special mode observations in fall 2010, the average background in orbits 99, 100, and 101 was $0.001 \mathrm{cnts} \mathrm{s}^{-1}$ as well, with a standard deviation of $0.0007 \mathrm{cnts} \mathrm{s}^{-1}$.

The special mode observations in fall 2010 (center energy $=10 \mathrm{eV}$ ) and in fall 2011 (center energy $=32 \mathrm{eV}$ ) are the two only occasions when integration times per pixel were principally sufficient to detect the oxygen fall peak. In fall 2010, a cluster of six adjacent pixels with enhanced count rates (see the blue pixel at spin angle $=120^{\circ}$ in the bottom left panel of Fig. 10) occurred at the place where we would expect the fall peak (see Fig. 5). The 8 counts in the brightest pixel correspond to $0.0021 \mathrm{cnts} \mathrm{s}^{-1}$ and would thus agree with the expected magnitude of the fall peak of $0.0018 \pm 0.0007 \mathrm{cnts} \mathrm{s}^{-1}$ if we take into account the background of 0.001 counts $\mathrm{s}^{-1}$. However, a similar enhancement is also observed around spin angle $80^{\circ}$ in the bottom left panel of Fig. 10. The main argument against an ISN origin of these clusters in fall 2010 is the absence of a similar enhancement one year later in orbits 143 and 144 (same ecliptic longitude as orbits 98 and 99). In fall 2011, IBEX observed neutrals in energy bin 2, which covers the expected energy (38 eV) of the oxygen bulk flow. The non-detection in fall 2011 is illustrated by the line profiles in Fig. 11. The 
difference between the two profiles is due to different approaches in data culling. The dataset with the lower count rates (shown by the black " $\mathrm{x}$ " symbols) is the default data selection with a restrictive a priori time selection. The error bars were derived from Poisson statistics. They are usually $50 \%-100 \%$ of the values themselves because only a few counts were registered during the restricted observation time. We therefore experimented with longer integration times, abandoning the requirement that IBEX must be outside the bow shock of the magnetosphere. The results are shown as red triangles in Fig. 11. To limit the contamination of the magnetosphere, we excluded data blocks where the number of oxygen counts or the number of hydrogen counts exceeded the 3- $\sigma$ limit expected for quiet time periods (see Equation 2). Nevertheless, the less restrictive dataset shows a significantly higher average count rate (the red dotted line at $0.001 \mathrm{cnts} \mathrm{s}^{-1}$ ) than the average level of $0.0005 \mathrm{cnts} \mathrm{s}^{-1}$ obtained for the nominal data set (black dotted line). For the less selective data set, the count rates also increase toward the poles, which are closer to the heavily contaminated ram hemisphere. We notice only two single spin angles $\left(97^{\circ}\right.$ and $\left.127^{\circ}\right)$ in the center of the anti-ram hemisphere $\left(90^{\circ} \pm 45^{\circ}\right)$ where both data sets yield count rates notably higher than the background levels. Since there is no group of neighboring pixels with enhanced signals we consider the difference between these single incidences and the average signal level as the upper limit of the oxygen fall signal.

In summary, the IBEX observations yielded an upper limit of $0.001 \mathrm{cnts} \mathrm{s}^{-1}$ for the ISN oxygen fall peak in the anti-ram hemisphere in fall 2011 (see Fig. 11). This agrees with the expected fall peak magnitude of $0.0008 \mathrm{cnts} \mathrm{s}^{-1}$. Unfortunately, the special mode data at $10 \mathrm{eV}$ in fall 2010 and in fall 2011 with a center energy of $32 \mathrm{eV}$ are unique in the IBEX-Lo database so far. Observations in nominal mode with an eight times shorter integration time are principally insufficient to detect the oxygen fall peak even in the absence of magnetospheric contamination due to the very low count statistics. Special oxygen mode measurements were not repeated in later fall seasons. Detecting atoms heavier than helium 
requires a high post-acceleration voltage. We had, however, to reduce the post-acceleration voltage of IBEX-Lo from $16 \mathrm{kV}$ to $7 \mathrm{kV}$ in summer 2012. Early attempts to increase high voltage again did not succeed and IBEX-Lo is currently running at $7 \mathrm{kV}$. We therefore cannot check if the potential signal appears at the same place on consecutive years.

\section{Conclusions}

The fall signal of neutral interstellar helium from anti-ram directions was not observed with IBEX-Lo. This non-detection cannot be explained by statistical limitations or by a local background masking the signal. It implies that the lower energy threshold for helium sputtering hydrogen lies between 25 and $30 \mathrm{eV}$, which agrees with SRIM sputtering simulations. This lower limit must be taken into account for future comparisons between models and IBEX maps at any region in the sky, especially for the Warm Breeze (Sokót et al. 2015b).

The fall peak of interstellar oxygen, on the other hand, could in principle be observed with IBEX-Lo if the instrument is run in a special mode where the integration time per pixel is eight times longer than in the nominal mode. Even so, the two attempts in 2010 and 2011 did not yield an unambiguous detection because the background due to magnetospheric contaminations in October is usually as large as the expected signal strength. The upper

limit derived from the best observation window in fall 2011 is $0.001 \mathrm{cnts} \mathrm{s}^{-1}$ in energy bin 2 from 21 to $43 \mathrm{eV}$, which agrees with predictions.

Acknowledgements. We thank all of the outstanding men and women who have made the IBEX mission such a wonderful success. A.G. and P.W. thank the Swiss National Science foundation for financial support. M.B., M.A.K., J.M.S., and P.S. were supported by Polish National Science Center grant 2012-06-M-ST9-00455. J.P., H.K., E.M., N.S., S.A.F., 
and D.J.M. were supported by the NASA Explorer program as a part of the IBEX mission. 


\section{REFERENCES}

Bzowski, M., Kubiak, M. A., Möbius, E., et al. 2012, ApJS, 198, 12

Bzowski, M., Sokół, J. M., Kubiak, M. A., \& Kucharek, H. 2013, A\&A, 557, A50

Fuselier, S. A., Allegrini, F., Bzowski, M., et al. 2012, ApJ, 754, 14

Fuselier, S. A., Bochsler, P., Chornay, D., et al. 2009, Space Science Reviews, 146, 117

Galli, A., Wurz, P., Fuselier, S. A., et al. 2014, ApJ, 796, 9

Kubiak, M. A., Bzowski, M., Sokół, J. M., et al. 2013, A\&A, 556, A39

Kubiak, M. A., Bzowski, M., Sokół, J. M., et al. 2014, ApJS, 213, 29

Kucharek, H., et al. 2015, submitted to this issue

McComas, D.J., Allegrini, F., Bochsler, P., et al. 2009, Space Science Reviews, 146, 11

McComas, D. J., Bzowski, M., Frisch, P., et al. 2010, J. Geophys. Res., 115, A09113, doi:10.1029/2010JA015569

McComas, D. J., Dayeh, M. A., Allegrini, F., et al. 2012, ApJS, 203, 1

McComas, D. J., Dayeh, M. A., Funsten, H. O., et al. 2011, J. Geophys. Res., 116, A02211, doi:10.1029/2010JA016138

Möbius, E., Bochsler, P., Bzowski, M., et al. 2009, Science, 326, 969

Möbius, E., Bochsler, P., Bzowski, M., et al. 2012, ApJS, 198, 11

Park, J., Kucharek, H. et al. 2015, submitted to this issue

Petrinec, S. M., Dayeh, M. A., Funsten, H. O., et al. 2011, J. Geophys. Res., 116, A07203, doi:10.1029/2010JA016357 
Rodriguez Moreno, D. F., Wurz, P., Galli, A., et al. 2015, submitted to this issue

Rodriguez Moreno, D. F., Wurz, P., Saul, L., et al. 2013, A\&A, 557, A125

Saul, L., Bzowski, M., Fuselier, S. A., et al. 2013, ApJ, 767, 130

Schwadron, N. A., Möbius, E., Fuselier, S. A., et al. 2014, ApJS, 215, 13

Schwadron, N. A., Möbius, E., Kucharek, H., et al. 2013, ApJ, 775, 86

SILSO, World Data Center - Sunspot Number and Long-term Solar Observations, Royal Observatory of Belgium 2008-2014, International Sunspot Number Monthly Bulletin and online catalogue, http://www.sidc.be/SILSO/

Sokót, J. M., et al. 2015a, The Warsaw Test Particle Model (WTPM): modeling of the interstellar neutral helium flow measured by IBEX, submitted to this issue

Sokót, J. M., et al. 2015b, The interstellar He haze in the heliosphere: what can we learn?, submitted to this issue

Taglauer, E. 1990, Appl. Phys. A 51, 238-251

Wurz, P., Saul, L., Scheer, J., et al. 2008, Jou. Appl. Phys., 103, 054904

Wurz, P., Scheer, J., \& Wieser, M. 2006, e-Jou. Surf. Science Nanotechn., 4, 394-400

Ziegler, J. F., Biersack, J., \& Littmark, U. 1985, The Stopping and Range of Ions in Matter, (Pergamon Press) 


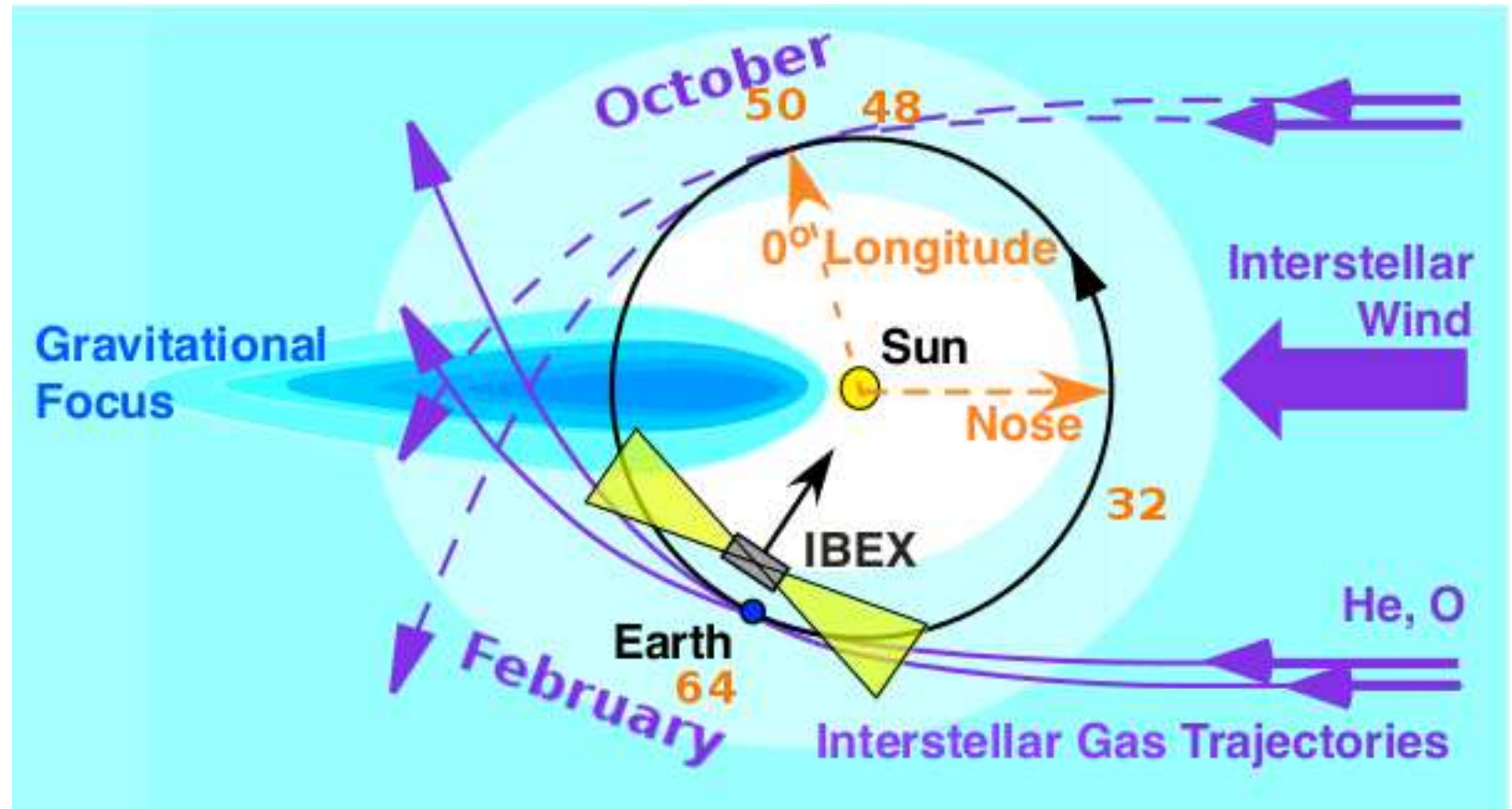

Fig. 1. - Sketch of the interstellar gas flow distribution for $\mathrm{He}$ in the inner heliosphere along with ISM bulk flow trajectories that are observable with IBEX at 1 AU. The solid line denotes the trajectory of the spring signal seen from ram- directions, the dashed line is the trajectory of neutral helium arriving at IBEX from anti-ram direction in October. The orange figures indicate orbit numbers for the year 2009/2010; from June until September (orbits 32 to 48), IBEX mostly is inside Earth's magnetosphere and no useful observations can be obtained. Figure adapted from Möbius et al. (2012). 


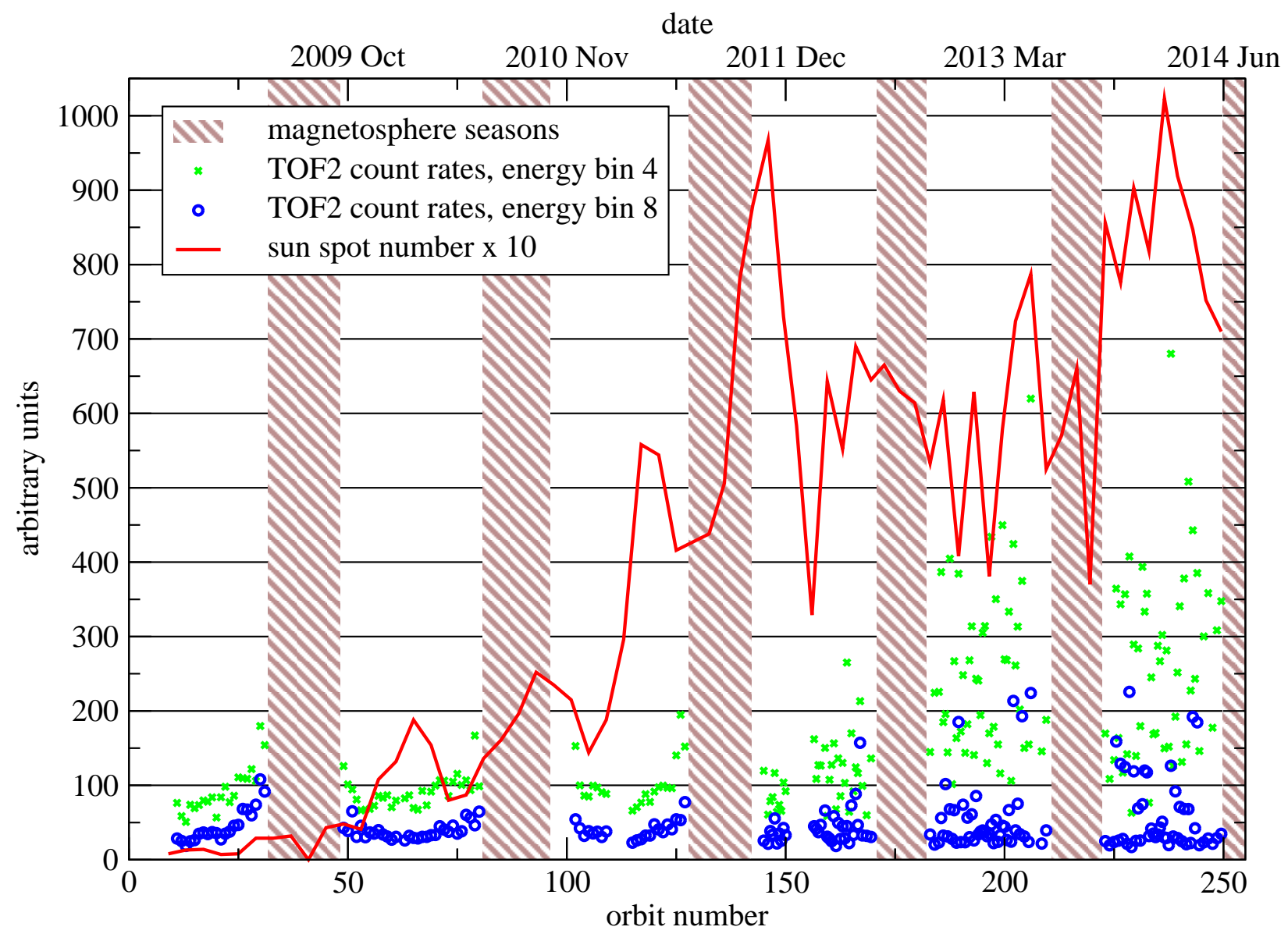

Fig. 2.- Time-series of uncorrelated TOF2 count rates in IBEX-Lo for the first six years (upper axis shows the date, lower $\mathrm{x}$-axis shows orbit number) of the mission versus series of sun spot numbers. The TOF2 count rates are averages per IBEX orbit, the sun spot numbers are the monthly averages provided by SILSO, World Data Center (2008). The shaded areas denote the data gaps from June until September when IBEX is inside Earth's magnetosphere. Energy bin 4 has a center energy of $110 \mathrm{eV}$ for hydrogen, energy bin 8 has a center energy of $1821 \mathrm{eV}$. Both the scatter and the average level of TOF2 count rates increase with time as solar activity increases. Moreover, the low energies are more sensitive to magnetospheric contamination. 


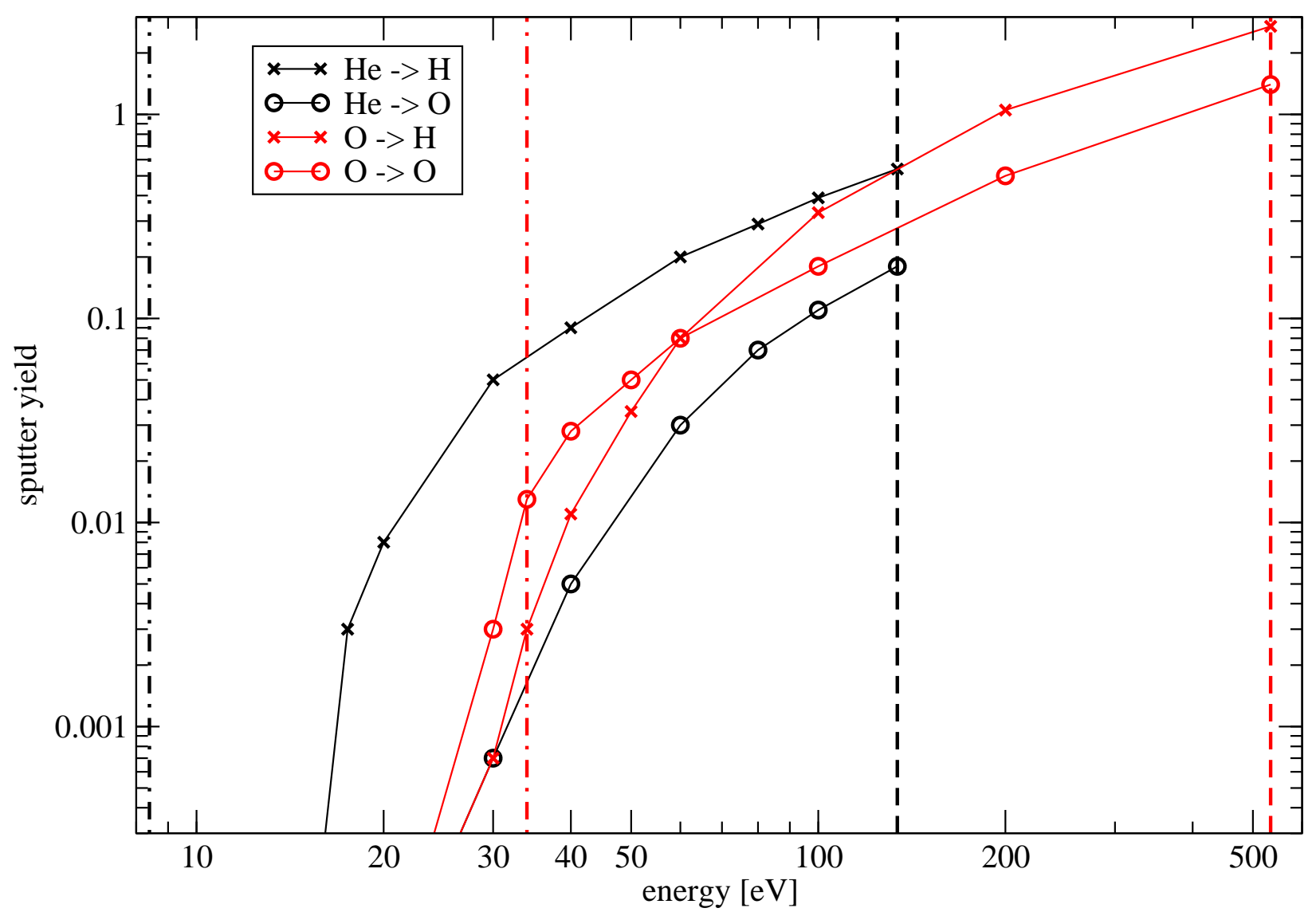

Fig. 3.- Sputter yield (ratio of sputtered versus impacting atoms) simulated with the SRIM software for helium and oxygen atoms hitting the conversion surface of IBEX-Lo at $15^{\circ}$ and sputtering hydrogen and oxygen atoms. The dashed lines indicate the relative velocity of ISN helium (black) and oxygen (red) for ram observations in the spring season, the corresponding dash-dotted lines show the velocities of the ISN species for anti-ram observations. 


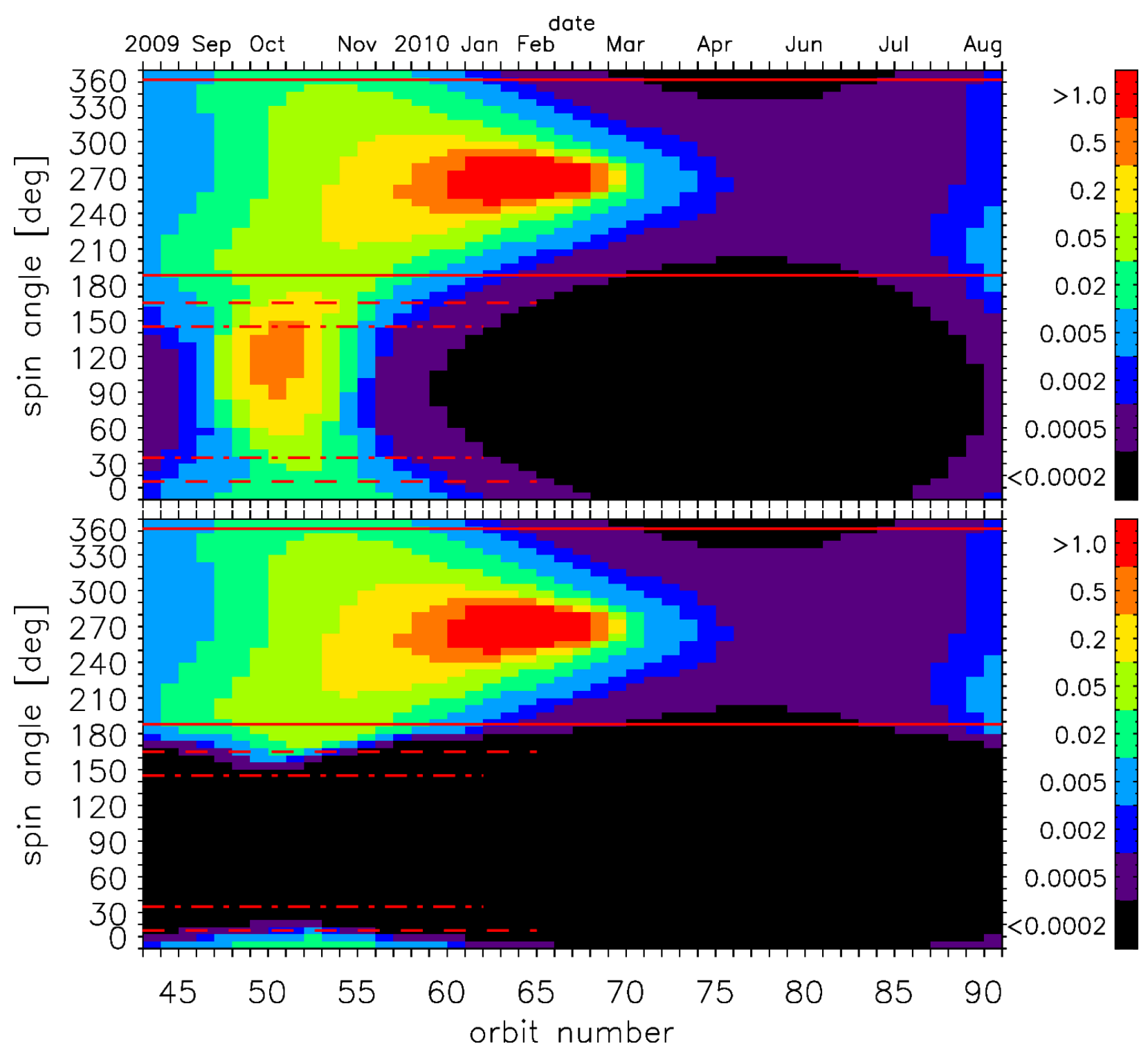

Fig. 4. - Predicted count rates of sputtered hydrogen due to ISN helium, simulated as a combination of the ISN primary population and of the Warm Breeze (taken from Fig. 7 in Sokót et al. (2015b)). The count rates were scaled in such a way that the spring peak in the ram hemisphere (spin angles around $270^{\circ}$ ) reaches the observed $23 \mathrm{cnts} \mathrm{s}^{-1}$. If helium of any energy could sputter hydrogen atoms, the fall peak in the anti-ram hemisphere should also be clearly visible (upper panel). In the realistic case where no hydrogen atoms are sputtered by helium below $19 \mathrm{eV}$ in the spacecraft reference frame, the fall peak cannot be observed (lower panel). Red lines denote energy limits relevant for IBEX-Lo observations (solid lines: limits for energy bin 2, dashed lines: energy bin 1, dash-dotted lines: limits for the lowest energy bin 0.5 ). 


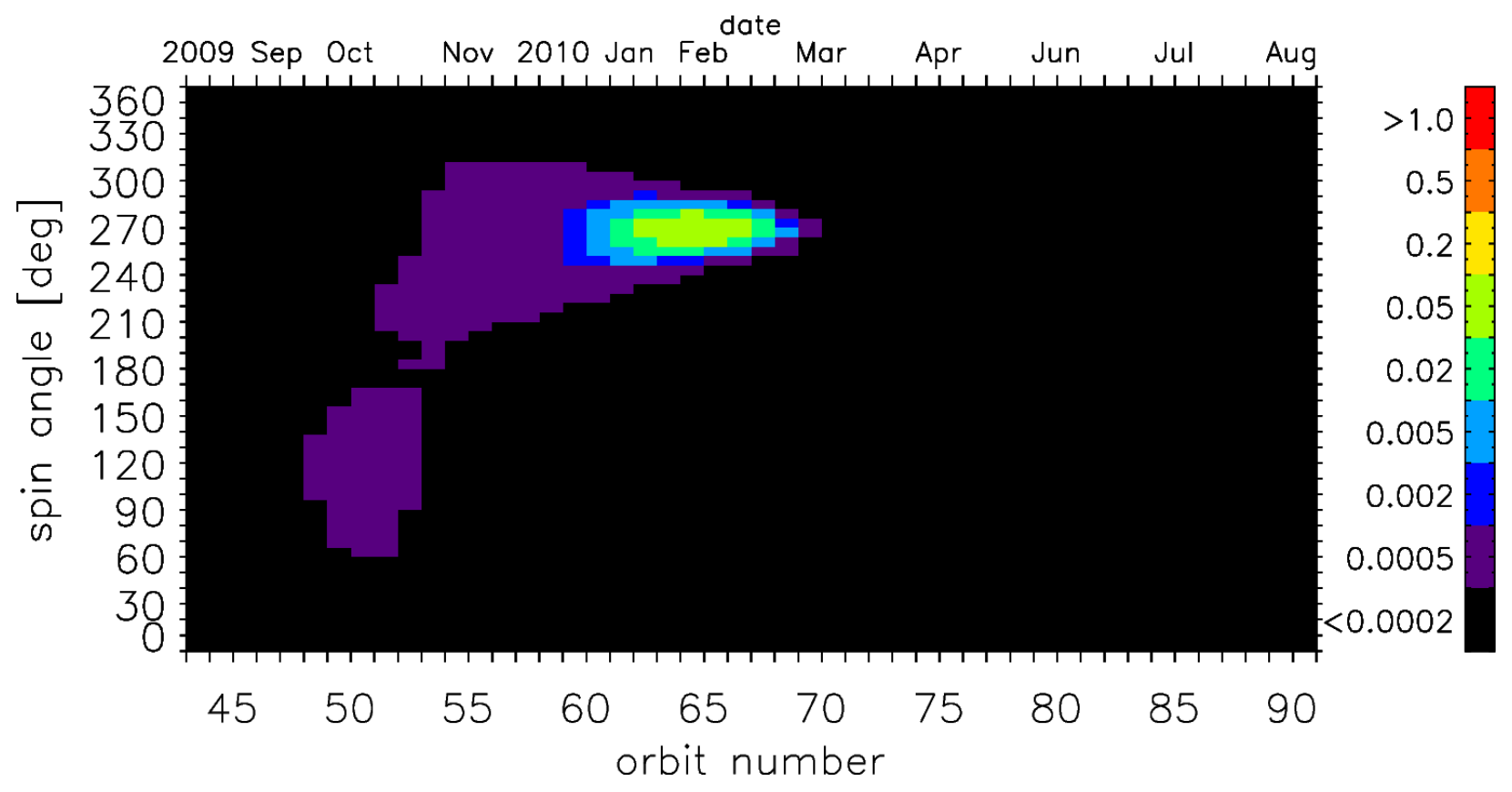

Fig. 5.- Count rates of ISN oxygen based on simulations of ISN helium under the assumption that the particle trajectories in the heliosphere are identical for both species; same format as Fig. 4. The main peak in the ram hemisphere (spin angles $180^{\circ}-360^{\circ}$ ) is the signal seen in February and March. Blue pixels (0.0015 to $0.003 \mathrm{cnts} \mathrm{s}^{-1}$ ) denote the statistical detection limit for the nominal mode, i.e., one single count per orbit. Purple pixels $(0.0002$ to $0.0015 \mathrm{cnts} \mathrm{s}^{-1}$ ) denote the detection limit for the special fall mode with an eight times longer integration time. 

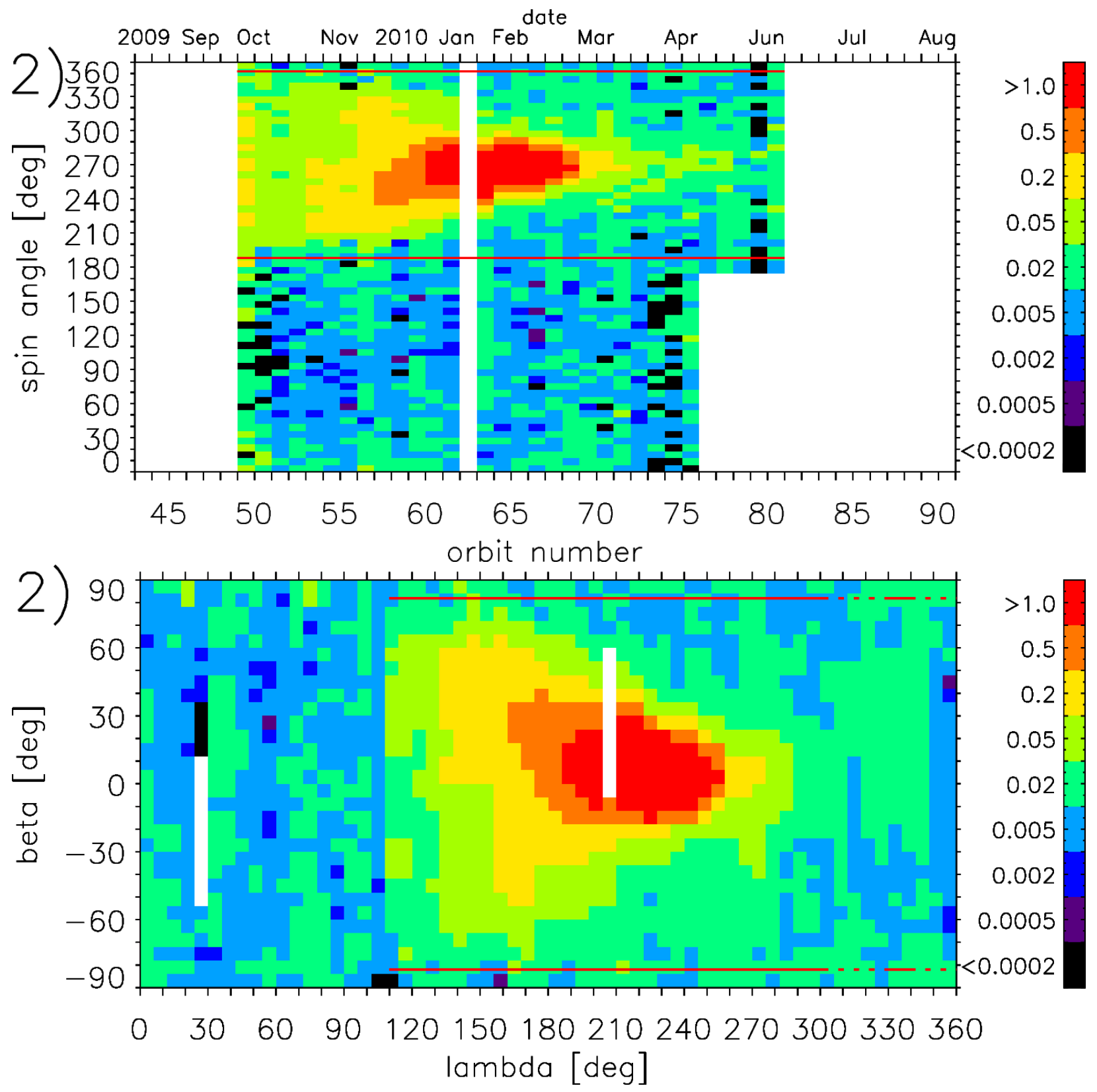

Fig. 6.- Maps of hydrogen count rates measured in energy bin 2 from 2009 October 13 until 2010 June 10. The upper plot shows raw IBEX coordinates with spin angle sectors versus orbits (orbits 49-80). Spin angles between $180^{\circ}$ and $360^{\circ}$ correspond to ram measurements where the intense sputter signal from ISN helium is observed around orbit 64 in February. The lower panel shows the same measurements (without orbit 49) organized in ecliptic coordinates. The spring signal occurs at $\lambda_{e c l}=225^{\circ}$, pixels between $290^{\circ}<\lambda_{\text {ecl }}<360^{\circ}$ are the average of ram and anti-ram measurements. The lines in the ram hemisphere contain the expected range of ISN helium energetic enough to create a signal in this energy bin. 


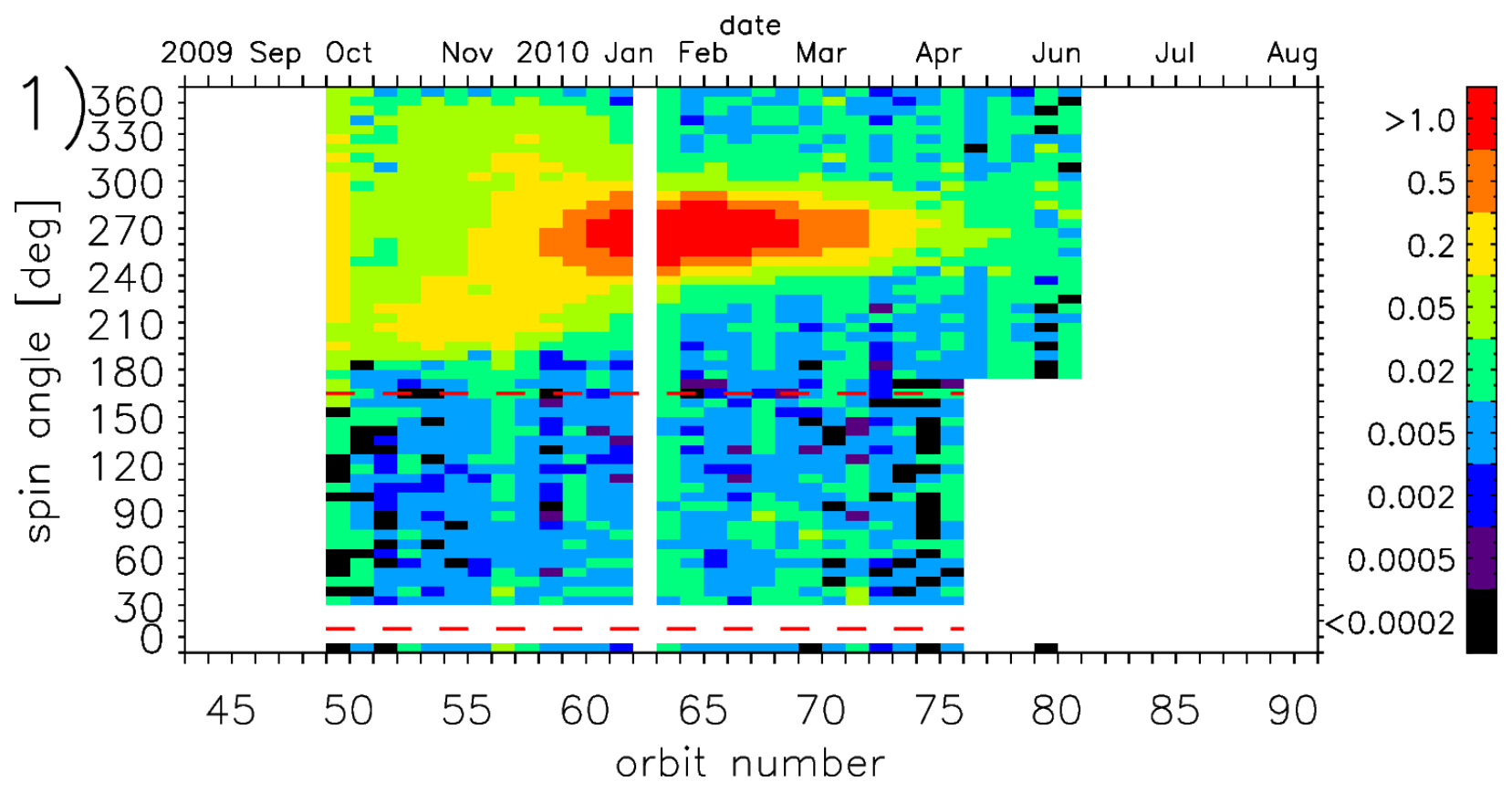

Fig. 7.- Map of hydrogen count rates measured in energy bin 1 from 2009 October 13 until 2010 June 10. The plot shows the IBEX reference frame with spin angle sectors versus orbits (orbits 49-80), same format as the upper panel of Fig. 6. The dashed lines indicate the expected cut-off of the ISN signal. The white vertical strip at orbit 62 , for which no data are available, is due to an on-board computer glitch. 

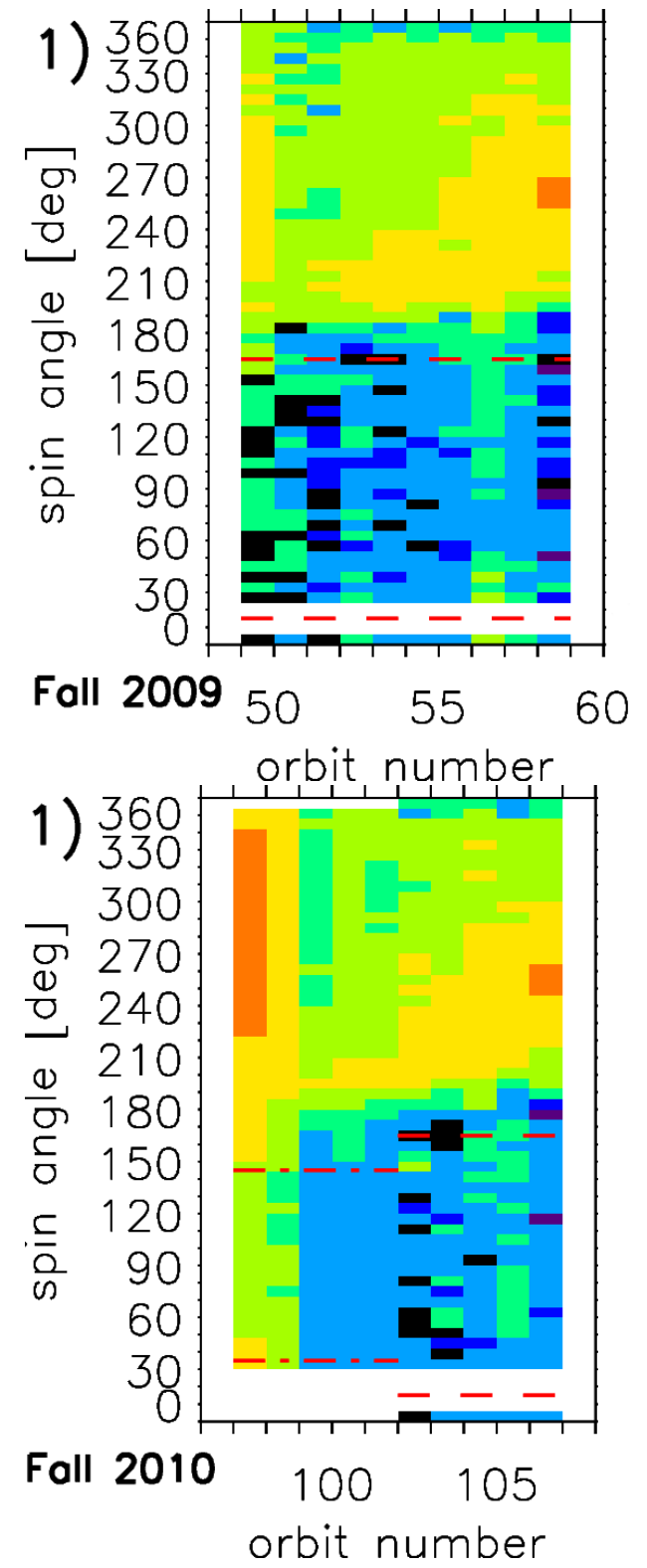

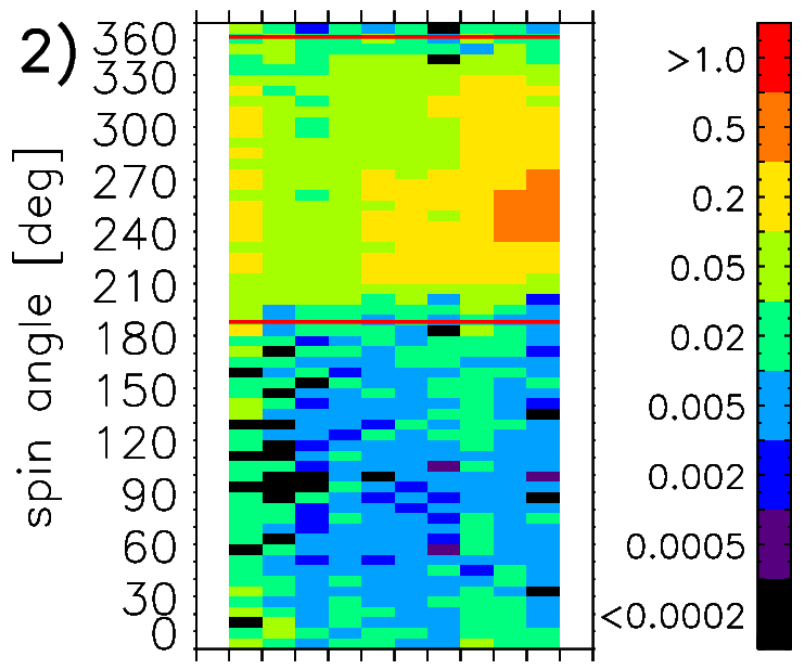

Foll $200950 \quad 55 \quad 60$

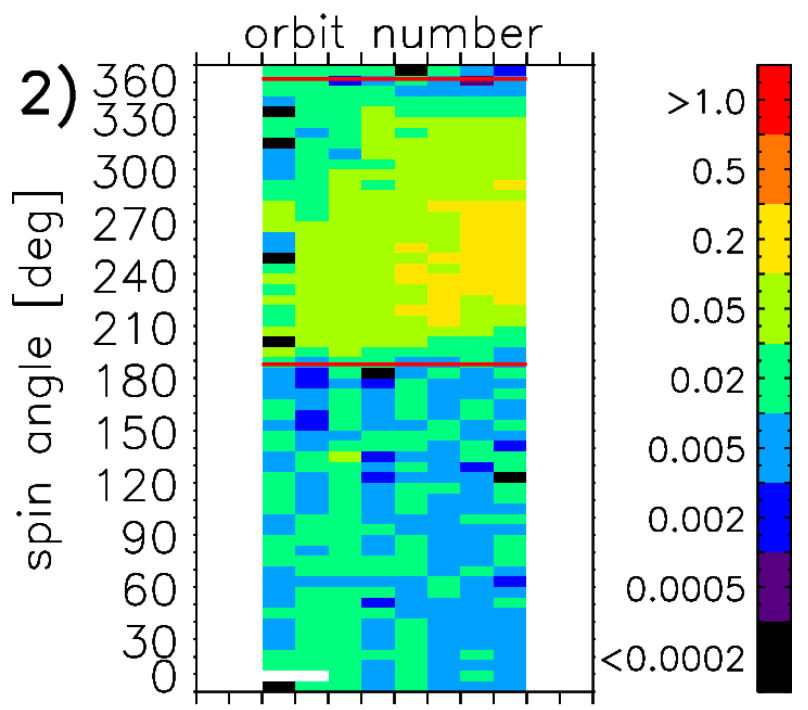

Foll 2011

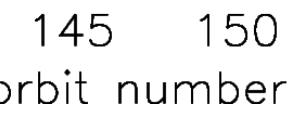

Fig. 8.- Maps of hydrogen count rates for the fall season from October to December, same format as the upper panel of Fig. 6. Top left: fall season 2009, energy bin 1 (center energy $=15 \mathrm{eV}$ ), top right: fall season 2009, energy bin 2 (center energy $=29 \mathrm{eV})$. Bottom left: fall season 2010, during orbits 97-101 the instrument was run in a special mode with the center energy at $10 \mathrm{eV}$ and eight times longer integration time, for the subsequent orbits the instrument was run in nominal energy bin 1. Bottom right: fall season 2011, energy bin 2, during orbits 143-144 the sensor was run in a special mode with eight times longer integration time. The red lines indicate the expected cut-off of the ISN helium signal in the respective energy bin. 

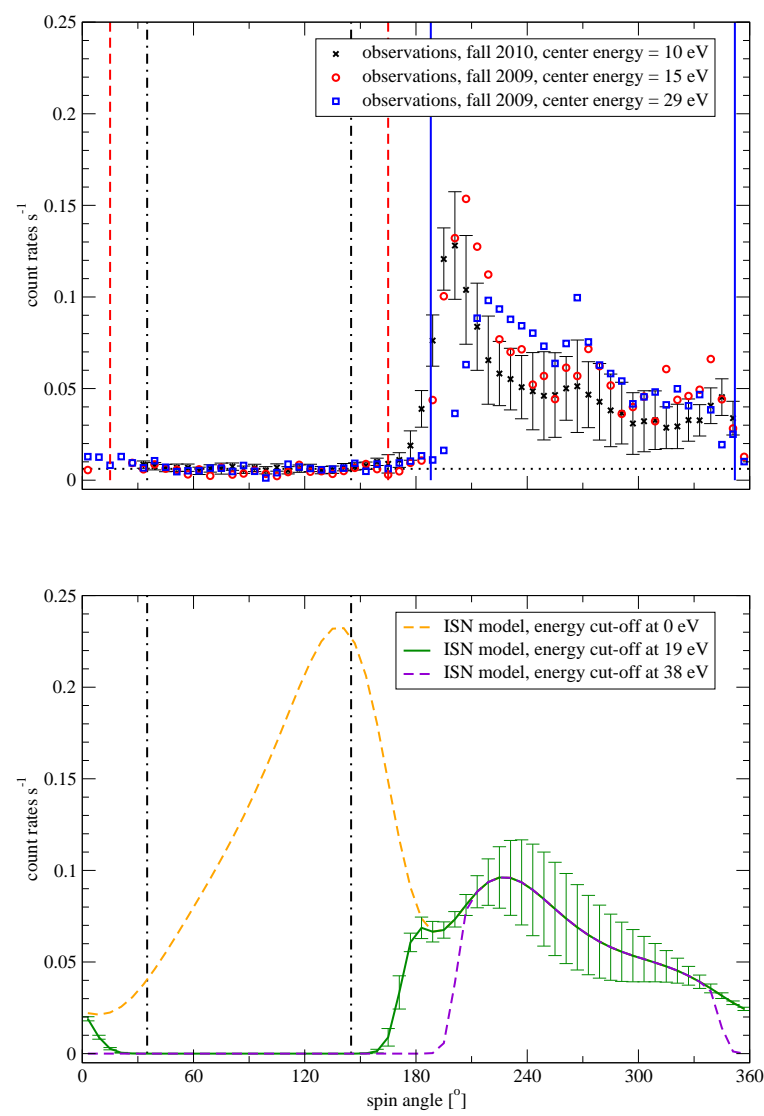

Fig. 9.- Latitudinal profiles of measured (top) and expected (bottom) hydrogen count rates due to ISN helium during fall season. The red circles (energy bin 1) and the blue squares (energy bin 2) show observations during nominal mode in fall 2009 (orbits 51-53), the black " $\mathrm{x}$ " denote the measured count rates during the special mode with center energy at $10 \mathrm{eV}$ for 2010 October 27 to November 16 (orbits 99-101). Between the anti-ram direction (spin angle $90^{\circ}$ ) and the vertical line for a given energy bin, the sensor principally cannot detect any ISN signal because of the finite energy range of the respective bin. The black dotted line denotes the derived background level of $0.0062 \mathrm{cnts} \mathrm{s}^{-1}$ during special mode measurements. The model results, averaged for orbits 51-53, are shown as follows: the orange dashed line is the model profile predicted with no energy threshold for sputtering. Blue solid line: model profile with a lower cut-off at $19 \mathrm{eV}$, purple dashed line: model profile with a $38 \mathrm{eV}$ cut-off. 

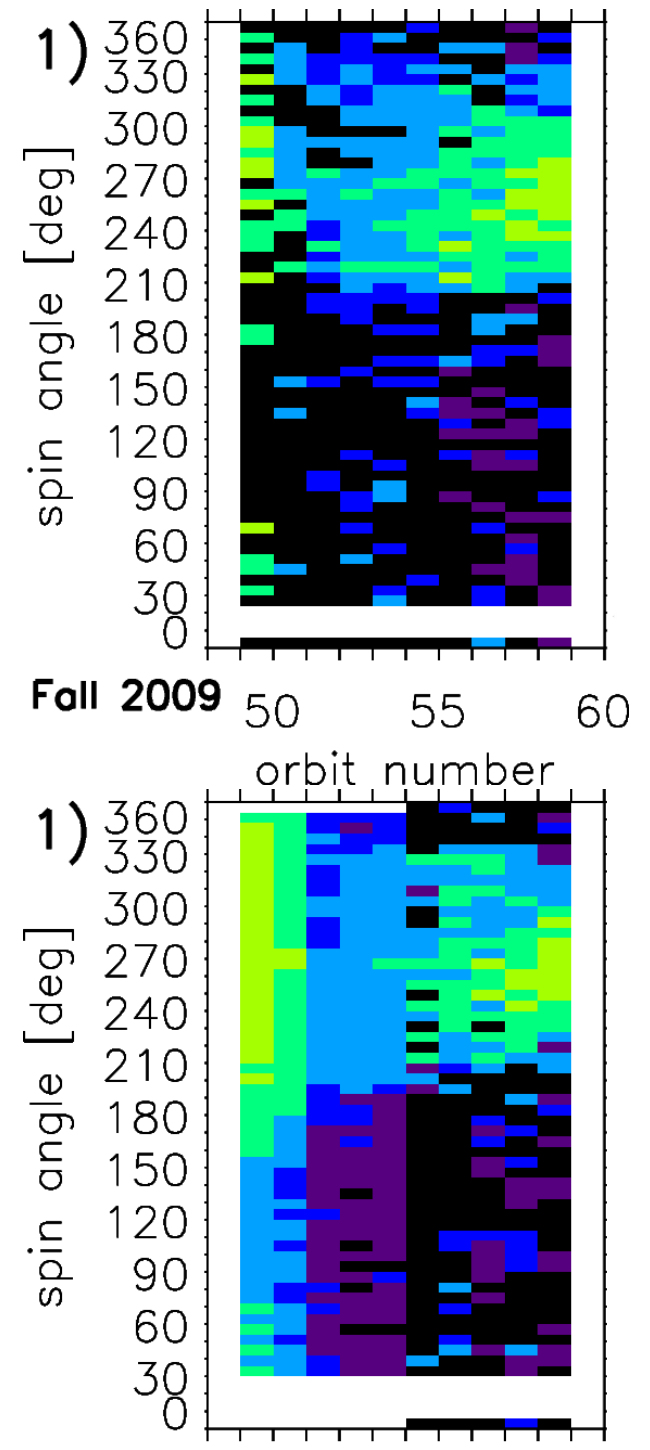

\section{Foll $2010 \quad 100 \quad 105$ orbit number}

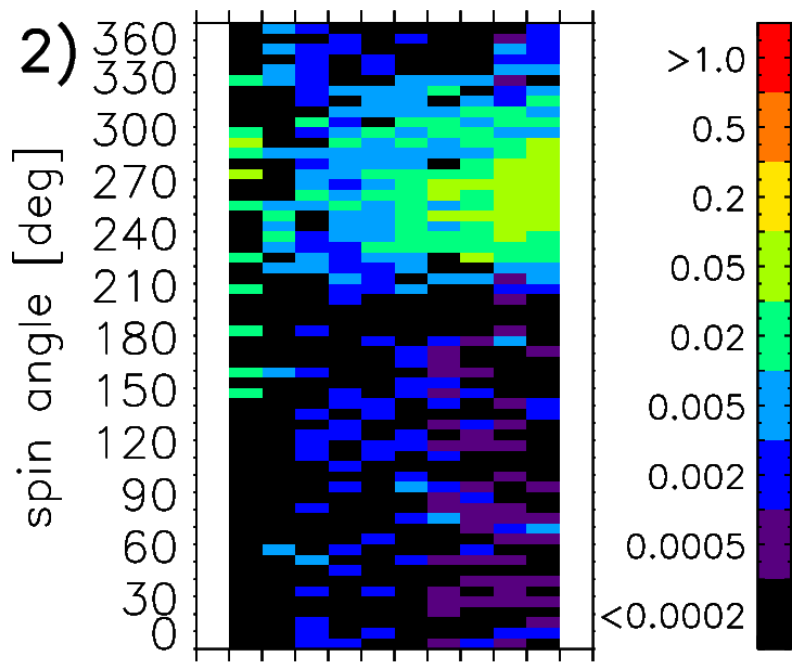

Foll $200950 \quad 55 \quad 60$

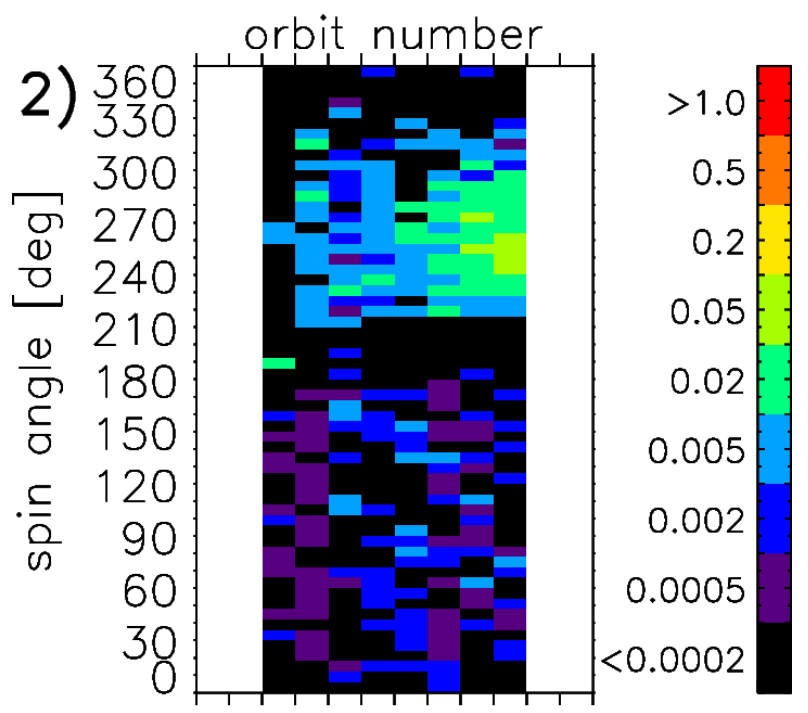

Foll 2011

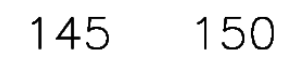

orbit number

Fig. 10.- Maps of oxygen count rates for the fall season from October to December, same format as Fig. 8 for hydrogen count rates. Top left: fall season 2009, energy bin 1 (center energy $=15 \mathrm{eV})$, top right: fall season 2009, energy bin $2($ center energy $=29 \mathrm{eV})$. Bottom left: fall season 2010, for orbits 97-101 IBEX-Lo was run in a special fall mode with center energy at $10 \mathrm{eV}$, for the subsequent orbits the instrument was run at nominal energy bin 1. Bottom right: fall season 2011, energy bin 2. During orbits 143-144 the sensor was run in a special mode with eight times longer integration time. 


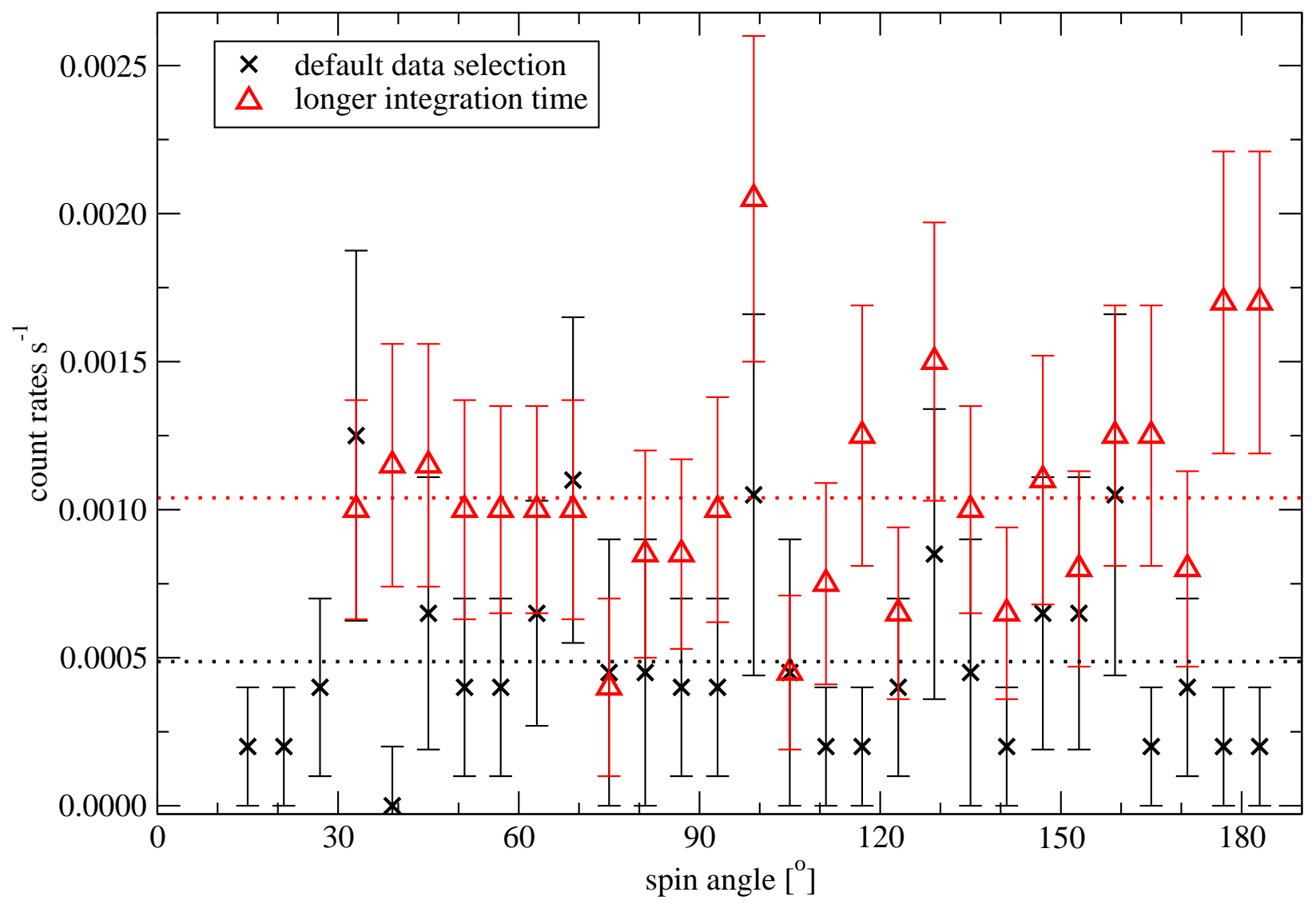

Fig. 11.- Oxygen count rates in energy bin 2, averaged over orbits 143 and 144 during the special mode observations in fall 2011. The count rates are organized according to IBEX spin angle for the anti-ram hemisphere $\left(0^{\circ}\right.$ corresponding to South pole and $180^{\circ}$ to the North pole of the ecliptic). Averages are indicated as dotted lines, the data plotted with black " $\mathrm{x}$ " were obtained by the nominal restrictive data selection, the data plotted with red triangles resulted from longer integration times and thus potentially higher magnetospheric contamination. 
Table 1: Table of IBEX-Lo energies. Top: for hydrogen ENAs (also relevant for helium observations), bottom: for oxygen ENAs. The energies for $\mathrm{H}$ and $\mathrm{O}$ differ because the energy loss on the conversion surface of the sensor depends on the atom species. The last column lists the velocity of a helium (top) and oxygen (bottom) atom that corresponds to the center energy.

\begin{tabular}{|c|c|c|c|c|c|}
\hline name & species & lower boundary $(\mathrm{eV})$ & center energy $(\mathrm{eV})$ & upper boundary $(\mathrm{eV})$ & $v\left(\mathrm{~km} \mathrm{~s}^{-1}\right)$ \\
\hline bin 0.5 & $\mathrm{H}, \mathrm{He}$ & 7 & 10 & 15 & 22.0 \\
\hline bin 1 & $\mathrm{H}, \mathrm{He}$ & 11 & 15 & 21 & 26.9 \\
\hline bin 2 & $\mathrm{H}, \mathrm{He}$ & 20 & 29 & 41 & 37.4 \\
\hline bin 3 & $\mathrm{H}, \mathrm{He}$ & 39 & 55 & 77 & 51.5 \\
\hline bin 4 & $\mathrm{H}, \mathrm{He}$ & 78 & 110 & 155 & 72.9 \\
\hline bin 5 & $\mathrm{H}, \mathrm{He}$ & 150 & 209 & 296 & 100.4 \\
\hline bin 6 & $\mathrm{H}, \mathrm{He}$ & 301 & 439 & 608 & 145.5 \\
\hline bin 7 & $\mathrm{H}, \mathrm{He}$ & 605 & 872 & 1215 & 205.1 \\
\hline bin 8 & $\mathrm{H}, \mathrm{He}$ & 1349 & 1821 & 2624 & 294.4 \\
\hline bin 0.5 & $\mathrm{O}$ & 7 & 10 & 15 & 11.0 \\
\hline bin 1 & $\mathrm{O}$ & 11 & 15 & 21 & 13.5 \\
\hline bin 2 & $\mathrm{O}$ & 21 & 32 & 43 & 19.7 \\
\hline bin 3 & $\mathrm{O}$ & 42 & 65 & 87 & 28.0 \\
\hline bin 4 & $\mathrm{O}$ & 84 & 135 & 178 & 40.4 \\
\hline bin 5 & $\mathrm{O}$ & 170 & 279 & 367 & 58.0 \\
\hline bin 6 & $\mathrm{O}$ & 371 & 601 & 791 & 85.2 \\
\hline bin 7 & $\mathrm{O}$ & 742 & 1206 & 1582 & 120.6 \\
\hline bin 8 & $\mathrm{O}$ & 1444 & 2361 & 3097 & 168.8 \\
\hline
\end{tabular}


Table 2: Ubiquitous background hydrogen count rates in the lower energies of IBEX-Lo derived from all five years of data. The background was quantified by demanding that the heliospheric ENA signal in the solar inertial reference frame (between $300^{\circ}$ and $360^{\circ}$ ecliptic longitude) should be equal for ram and anti-ram observations after background subtraction. The oxygen background count rates are ten times lower for energy bins 1 to 5 .

\begin{tabular}{llr}
\hline energy bin & center energy & background count rate in s ${ }^{-1}$ \\
\hline 1 & $0.015 \mathrm{keV}$ & $0.0098 \pm 0.0025$ \\
2 & $0.029 \mathrm{keV}$ & $0.0089 \pm 0.0020$ \\
3 & $0.055 \mathrm{keV}$ & $0.0118 \pm 0.0015$ \\
4 & $0.110 \mathrm{keV}$ & $0.0113 \pm 0.0015$ \\
5 & $0.209 \mathrm{keV}$ & $0.0056 \pm 0.0010$ \\
6 & $0.439 \mathrm{keV}$ & $0.0008 \pm 0.0008$ \\
7 & $0.872 \mathrm{keV}$ & 0.0 \\
8 & $1.821 \mathrm{keV}$ & 0.0 \\
\hline
\end{tabular}


Table 3: Maximum spring peak in 2010 (measured in orbit 64) caused by ISN helium and oxygen, in energy bins 1-6. Part of the measured $\mathrm{H}$ signal in the two lowest energy bins is caused by true hydrogen, and the majority of the measured $\mathrm{O}$ signal in energy bins 1-4 is caused by ISN helium sputtering oxygen atoms.

\begin{tabular}{lrr}
\hline energy bin & $\mathrm{H}$ signal $\left(\mathrm{cnts} \mathrm{s}^{-1}\right)$ & $\mathrm{O}$ signal $\left(\mathrm{cnts} \mathrm{s}^{-1}\right)$ \\
\hline 1 & 18.5 & 3.4 \\
2 & 21.3 & 5.3 \\
3 & 22.6 & 4.6 \\
4 & 3.4 & 0.4 \\
5 & 0 & 0.05 \\
6 & 0 & 0.08 \\
\hline
\end{tabular}

